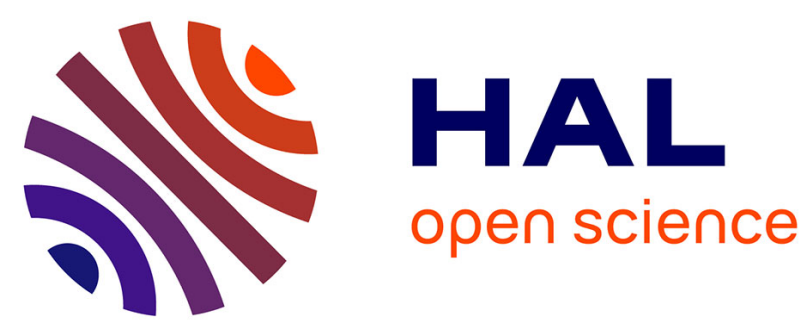

\title{
Distributed Minimum Euclidean Distance based Precoding for Wireless Sensor Network
}

Viet-Hoa Nguyen, Charlotte Langlais, Baptiste Vrigneau, Olivier Berder

\section{To cite this version:}

Viet-Hoa Nguyen, Charlotte Langlais, Baptiste Vrigneau, Olivier Berder. Distributed Minimum Euclidean Distance based Precoding for Wireless Sensor Network. International Conference on Computing, Networking and Communications (ICNC), IEEE, Feb 2015, Anaheim, United States. hal01121146

\author{
HAL Id: hal-01121146 \\ https://hal.inria.fr/hal-01121146
}

Submitted on 27 Feb 2015

HAL is a multi-disciplinary open access archive for the deposit and dissemination of scientific research documents, whether they are published or not. The documents may come from teaching and research institutions in France or abroad, or from public or private research centers.
L'archive ouverte pluridisciplinaire HAL, est destinée au dépôt et à la diffusion de documents scientifiques de niveau recherche, publiés ou non, émanant des établissements d'enseignement et de recherche français ou étrangers, des laboratoires publics ou privés. 


\title{
Distributed Minimum Euclidean Distance based Precoding for Wireless Sensor Network
}

\author{
Viet-Hoa NGUYEN ${ }^{1 *}$, Olivier BERDER ${ }^{1 *}$, Charlotte LANGLAIS ${ }^{2 \#}$, Baptiste VRIGNEAU $^{1 *}$ \\ ${ }^{1}$ IRISA/INRIA, Université de Rennes 1-ENSSAT, 6 rue de Kerampont - BP 80518 - F22305 Lannion, France \\ ${ }^{2}$ Lab-STICC, Telecom Bretagne, Technople Brest-Iroise - CS 83818 - 29238 Brest Cedex 3 - France \\ * $\{$ viet-h.nguyen, olivier.berder, baptiste.vrigneau\}@irisa.fr, \#charlotte.langlais@ @elecom-bretagne.eu
}

\begin{abstract}
Among various cooperative techniques aiming to reduce power consumption for Wireless Sensor Network (WSN), we present a new approach, named distributed $\max -d_{\min }$ precoding (DMP). This protocol is based on the deployment of a virtual $2 \times 2$ max- $d_{\min }$ precoding over one source, one forwarding relay, both equipped with one antenna and a destination involving 2 antennas. In this context, two kinds of relaying, amplify and forward or decode and forward protocols, are investigated. The performance evaluation in terms of Bit-Error-Rate (BER) and energy efficiency will be compared with non cooperative techniques (SISO, SIMO) and the distributed space time block code (STBC) scheme. Our investigations show that the DMP takes the advantage in terms of energy efficiency from medium transmission distances.
\end{abstract}

\section{INTRODUCTION}

The important achievements in recent years allow wireless sensor networks (WSN) to be applied more widely in real life. The demand of a flexible network leads to the use of low-cost, low-power, small size wireless sensor nodes with the embedded batteries. An important objective in WSN is how to maintain long-term operations of the network without the interruption caused by the battery replacement. Aiming to design an energy efficient transmission scheme, MIMO (multiple-input multiple-output) technique can be proposed with the benefits from a spatial diversity gain. However, due to the limited size, MIMO technique cannot be deployed by the classical manner in WSN. The idea is that the sensor nodes can share their antennas to create a virtual MIMO system, called cooperative communication.

A well-known cooperative scheme has been proposed by Laneman with a signal forwarding through the relay, called a cooperative relay [1]. In other studies, the authors apply spacetime block coding via the deployment of virtual MIMO system by using the relay at both transmission and reception sides [2] [3]. Owning the diversity gain, these cooperative schemes provide significant improvement in transmission power consumption compared to single input single output (SISO) system or maximum ratio combining (MRC) over single input multiple output (SIMO) system. On the other hand, they consume more energy for the electrical circuit and data exchanging. At small distances, when the circuit consumption dominates the total power consumption, a non cooperative protocol like SISO or SIMO provides better energy efficiency than the cooperative ones. Conversely, the cooperative schemes take the advantage in the larger distances.
Thanks to multiple transmit and receive antennas, the MIMO precoding involves a closed-loop spatial multiplexing, which exploits the Channel State Information at Transmitter (CSIT) to deal with the impairments of the propagation channel. In point-to-point MIMO, various precoding designs adapted to different criteria, such as maximizing the output capacity, maximizing the received signal-to-noise ratio, maximizing the minimum singular value of the channel matrix, have been studied [4] [5]. In [6] [7], the authors have proposed the max $-d_{\min }$ precoder, which maximizes the minimum Euclidean distance between the received vectors. In terms of $\mathrm{BER}$, this precoder outperforms the others at the expense of a more complex structure. Moreover, with respect to orthogonal space-time block codes, this precoder allows for higher spectral efficiency thanks to spatial multiplexing.

The idea to deploy the MIMO precoding via the cooperative manner - called distributed precoding - is very promising in wireless communication. Although showing the potential, the study on the distributed precoding are still limited, especially in WSN. Most of existing distributed precoders are dedicated to cellular networks, and particularly the downlink via the coordination of multiple base stations. Some interesting structures can be cited such as weight sum rate maximization, leakage projected dirty paper coding, layered virtual SINR (signal to interference plus noise ratio) maximization [8] [9] [10]. Unlike WSN, in the cellular network, the energy efficiency constraint is no longer important, instead the data rate is focused. Another fundamental difference is the insignificant space limitation at the base station in the downlink, where multiple antennas can be easily deployed. The base station collaboration then enhances the spectral efficiency.

The distributed precoding in WSN exists mostly in the form of distributed beamforming [11] [12]. This distributed scheme shows an impressive SNR gain, but always suffers from the synchronization problem. Moreover, the survey on the energy efficiency issues have not been carefully investigated for this distributed scheme. The authors in [13] have presented also a distributed approach for WSN in using the max- $d_{\min }$ precoder and P-OSM (precoding for orthogonalized spatial multiplexing). But the investigations on the cooperation and relaying are very sparing and not clear. There are also the lack of a spectral-efficient representation due to the use of MIMO precoding (compared to the other cooperative schemes).

Thanks to the outstanding BER performance of max- $d_{\min }$ 
precoder, we introduce the distributed $\max -d_{\min }$ precoding as a cooperative scheme in WSN. Different to the study in [13], herein we propose to investigate different protocols using a cooperating node (Decode-Forward, Amplify-Forward). Regarding on the local data exchange of a cooperative scheme, we propose the transmission of a part of the precoded signal between the source and cooperative node aiming to enhance significantly the spectral efficiency. The energy efficiency is also studied with a global energy model containing the circuit and transmission consumption.

The rest of this paper is organized as follows: In Section II, we introduce the system description of our cooperative scheme and the principle of max- $d_{\min }$ precoding is described. In Section III, we propose the different protocols for relaying and local data exchanging. The power consumption model is investigated in the context of WSN in Section IV. The simulation results of our schemes are presented in Section V. Section VI finally concludes the paper.

\section{SYSTEM MODEL}

We consider a simplified network, described in Figure 1, involving three nodes: a source, a cooperative node and a destination. The source and the cooperative node are sensor nodes, equipped with a single antenna and constrained to low energy consumption. The destination is supposed to be an access point (AP) without power constraint and neither stringent complexity constraint, compared to the sensor nodes. By allowing two antennas at the access point, the network is equivalent to a virtual $2 \times 2$ MIMO system. An errorfree and delay-free feedback link provides the channel state information at the transmitter (CSIT). Thanks to this CSIT, MIMO precoding techniques can be applied to the virtual MIMO system to deal with the channel impairments such as fading, antenna correlation, shadowing. Among various criteria, we propose to apply the $\max -d_{\min }$ precoder due to its significant advantage in reducing BER. The max- $d_{\min }$ precoding will be deployed via the cooperative protocol by two transmission phases:

- Local data exchange: the source node shares its data with the cooperative node via the SISO channel $h$.

- Precoding transmission: thanks to the spatial multiplexing properties of the precoder, the source and the cooperative node transmit the precoded signals simultaneously to the access point via the virtual MIMO channel matrix $\mathbf{H}$.

To limit energy consumption at the sensor nodes, the precoding matrix $\mathbf{F}$ can be computed at the destination. Hence, the feedback link sends back the precoding matrix instead of the CSIT. Recently, a quantized max- $d_{\min }$ precoding based on limited codebook has been proposed [14] [13]. In this case, the low rate feedback link transmits the index of the precoding matrix that has been computed by the destination node.

\section{A. Max- $d_{\min }$ precoder}

In this subsection, we describe the design of a max- $d_{\min }$ precoder in a classic MIMO system. The transmission model

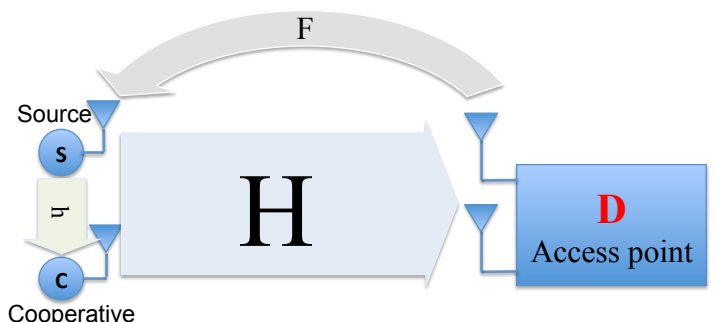

Fig. 1. System model

of the non cooperative $2 \times 2$ precoded system is given by

$$
\mathbf{y}=\mathbf{G H F} \mathbf{s}+\mathbf{G n}=\mathbf{G}_{d} \mathbf{H}_{v} \mathbf{F}_{d} \mathbf{s}+\mathbf{G}_{d} \mathbf{n}_{v},
$$

where $\mathbf{y}$ is the received vector, $\mathbf{s}$ is the transmitted vector of the modulated symbols, belonging to the QPSK constellation; $\mathbf{G}, \mathbf{H}, \mathbf{F}$ denote the decoder matrix, the MIMO channel matrix, the precoder matrix, respectively; and $\mathbf{n}$ is an additive white Gaussian noise (AWGN) vector. The virtual channel transformation allows to present the virtual channel in diagonal form $\mathbf{H}_{v}=\mathbf{G}_{v} \mathbf{H} \mathbf{F}_{v}=\operatorname{diag}\left(\sigma_{1}, \sigma_{2}\right) . \sigma_{1}, \sigma_{2}$ are the channel singular values in decreasing order, $\mathbf{n}_{v}$ is the virtual noise. Since the maximum likelihood detector is used at the receiver, the decoder matrix $\mathbf{G}_{d}$ has no impact on the performance, then it is assumed $\mathbf{G}_{d}=\mathbf{I}_{2}$. The max- $d_{\min }$ precoder $\mathbf{F}_{d}$ maximizes the minimum Euclidean distance between signal points at the reception side [6]:

$$
\mathbf{F}_{d}=\arg \max \left(\min _{\mathbf{s}_{k}, \mathbf{s}_{l} \in \mathcal{S}, \mathbf{s}_{k} \neq \mathbf{s}_{l}}\left\|\mathbf{H}_{v} \mathbf{F}_{d}\left(\mathbf{s}_{k}-\mathbf{s}_{l}\right)\right\|\right),
$$

where $\mathbf{s}_{k}, \mathbf{s}_{l}$ are two different transmitted vectors in the constellation $\mathcal{S}$. The max- $d_{\min }$ precoding solution depends on the modulation level, the number of antennas as well as channel properties [6] [7].

Let $\gamma=\operatorname{atan}\left(\frac{\sigma_{2}}{\sigma_{1}}\right)$ define the channel angle. For the $2 \times 2$ MIMO system with 2 QPSK multiplexed data streams, the max- $d_{\min }$ precoding matrix is given by [6]

- for $0 \leq \gamma \leq \gamma_{0} \approx 17.28^{\circ}$

$$
\mathbf{F}_{d}=\mathbf{F}_{r_{1}}=\left(\begin{array}{cc}
\sqrt{\frac{3+\sqrt{3}}{6}} & \sqrt{\frac{3-\sqrt{3}}{6}} e^{i \frac{\pi}{12}} \\
0 & 0
\end{array}\right)
$$

- for $\gamma_{0}<\gamma \leq 45^{\circ}$

$$
\mathbf{F}_{d}=\mathbf{F}_{\text {octa }}=\frac{1}{2}\left(\begin{array}{cc}
\cos \psi & 0 \\
0 & \sin \psi
\end{array}\right)\left(\begin{array}{cc}
1 & e^{i \frac{\pi}{4}} \\
-1 & e^{i \frac{\pi}{4}}
\end{array}\right)
$$

where $\psi=\operatorname{atan}\left(\frac{\sqrt{2}-1}{\tan \gamma}\right)$. The precoding matrix is then rewritten as $\mathbf{F}=\mathbf{F}_{v} \mathbf{F}_{d}$ under the power constraint trace $\left(\mathbf{F} \mathbf{F}^{*}\right)=1$. With $E\left[\mathbf{s s}^{*}\right]=E$, then $E\left[\operatorname{trace}\left((\mathbf{F s})(\mathbf{F s})^{*}\right)\right]=E$. It means the total energy in the precoding system for transmitting 2 symbols is only $E$, whereas in a SISO system this value is $2 E$ $\left(E\left[\operatorname{trace}\left(\mathbf{s s}^{*}\right)\right]=2 E\right)$. Therefore, to make a fair comparison in energy consumption, the transmit signal in the precoding system will be $\mathbf{x}=\sqrt{2} \mathbf{F s}$.

\section{B. Power allocation in cooperative systems}

In order to investigate the effects of the relay position in the cooperative schemes, we take into account the path loss explicitly in the transmission model. The distances $\mathrm{S} \rightarrow \mathrm{D}$, 
$\mathrm{S} \rightarrow \mathrm{C}, \mathrm{C} \rightarrow \mathrm{D}$ (Figure 1 ) are denoted by $d_{S D}, d_{S C}, d_{C D}$, respectively. Then the relative power gain of $\mathrm{S} \rightarrow \mathrm{C}$ and $\mathrm{C} \rightarrow \mathrm{D}$ links, with respect to $\mathrm{S} \rightarrow \mathrm{D}$ link, are given by $G_{S C}=\left(d_{S D} / d_{S C}\right)^{K}, G_{C D}=\left(d_{S D} / d_{C D}\right)^{K}$ [15], where $K$ is a path loss exponent, varying from 1.6 to 6 in indoor wireless channel. To have a fair comparison between various schemes, we assume the overall energy for each modulated symbol used in both local transmission $\left(E_{L}\right)$ and precoding transmission $\left(E_{D}\right)$ is $E$. It means $E_{L}=\beta E, E_{D}=(1-\beta) E$, where $\beta$ denotes the power allocation parameter.

\section{Distributed max $-d_{\min }$ PRECODING}

In this section, we propose two protocols, based on amplifyand-forward and decode-and-forward strategies, that distribute the max- $d_{\min }$ precoding over the source node and the cooperative node.

\section{A. Decode and Forward relaying (DMP-DF)}

The Decode and Forward scheme transmits 2 symbols within 3 time-slots. In the first and second time-slots, $s_{0}, s_{1}$ are sent symbol by symbol through a SISO channel, represented by $h$. Hence, the cooperative node receives

$$
\mathbf{y}_{c}=h \sqrt{E_{L} G_{S C}}\left[\begin{array}{ll}
s_{O} & s_{1}
\end{array}\right]^{T}+\mathbf{n}_{c},
$$

where $\mathbf{n}_{c}$ denotes the AWGN noise vector at the cooperative node. The received signal is then decoded to $s_{0}^{\prime}$ and $s_{1}^{\prime}$. The precoding signal is built as follows

$$
\begin{cases}F_{11} s_{0}+F_{12} s_{1} & \text { at the source node, } \\ F_{21} s_{0}^{\prime}+F_{22} s_{1}^{\prime} & \text { at the cooperative node, }\end{cases}
$$

where $\left.F_{i j}\right|_{i, j=1,2}$ are the elements of procoding matrix $\mathbf{F}$. In the third time-slot, both nodes transmit this signal simultaneously. Then, the destination receives

$$
\mathbf{y}=\mathbf{G H}\left(\begin{array}{c}
\sqrt{2 E_{D}}\left(F_{11} s_{0}+F_{12} s_{1}\right) \\
\sqrt{2 E_{D} G_{C D}}\left(F_{21} s_{0}^{\prime}+F_{22} s_{1}^{\prime}\right)
\end{array}\right)+\mathbf{G n},
$$

and applies a Maximum Likelihood detection. The precoding matrix $\mathbf{F}$ is designed only according to the channel $\mathbf{H}: \mathbf{F}=$ $\max -d_{\min }(\mathbf{H})$ [7] [6]. There is no need to estimate the SISO channel $h$ at the destination.

\section{B. Amplify and Forward relaying (DMP-AF)}

Instead of decoding the signal from the source node, the cooperative node amplifies it with a designed factor and forwards it to the destination. In this strategy, both channels $h$ and $\mathbf{H}$ have a significant influence on the system performance. Therefore, we propose two schemes with different uses of matrix $\mathbf{H}$ and $h$ for the design of the precoding matrix and the amplifying factor at the cooperative node. The first scheme, called factor multiplying, constructs the matrix $\mathbf{F}$ based on the channel $\mathbf{H}$. The effect of channel $h$ will be taken into account at the cooperative node by the amplifying factor. The second scheme, called channel customizing, transforms equivalently the received signal, bringing out a customized channel matrix containing both $\mathbf{H}$ and $h$. This scheme assumes the knowledge of $h$ and $\mathbf{H}$ at the destination.
Regarding the local data exchange between the source and the cooperative node, two configurations are carried out: 1) the transmission of the non precoded symbols on orthogonal channels, typically thanks to TDMA (Time division multiple access), 2) the transmission of a part of the precoded signal, i.e. a linear combination of the symbols. In this transmission phase, the signal from source node is not useful at the destination.

1) Factor multiplying: We design the precoding matrix according to the channel matrix $\mathbf{H}\left(\mathbf{F}=\max -d_{\min }(\mathbf{H})\right)$ and reduce the effect of channel $h$ by multiplying the received signal with an amplifying factor.

- Configuration 1: Local successive transmission (LST): $s_{0}$ and $s_{1}$ will be sent one by one to the cooperative node, according to TDMA. The cooperative node receives

$$
\mathbf{y}_{c}=\left(\begin{array}{l}
y_{c 0} \\
y_{c 1}
\end{array}\right)=\left(\begin{array}{l}
h \sqrt{E_{L} G_{S C}} s_{0}+n_{c 0} \\
h \sqrt{E_{L} G_{S C}} s_{1}+n_{c 1}
\end{array}\right) .
$$

At the cooperative node, the received signal is normalized by its energy, then precoded, and forwarded to the destination. At the same time, the source node also transmits its precoded signal to the destination. Then the received vector at the destination is written as

$$
\mathbf{y}=\mathbf{G H}\left(\begin{array}{c}
\sqrt{2 E_{D}}\left(F_{11} s_{0}+F_{12} s_{1}\right) \\
g_{1}\left(F_{21} y_{c 0}+F_{22} y_{c 1}\right)
\end{array}\right)+\mathbf{G n},
$$

where amplifying factor $g_{1}$ is chosen as [1]

$$
g_{1}=\frac{h^{*}}{|h|} \sqrt{\frac{2 E_{D} G_{C D}}{E_{L} G_{S C}|h|^{2}+N_{n_{c}}}},
$$

where $N_{n_{c}}$ is the noise variance at the cooperative node. The factor $g_{1}$ is aimed to reduce the effect of the AWGN noise, the channel $h[1]$ and prevent the power saturation at the cooperative node.

- Configuration 2: Local precoded transmission (LPT): Instead of transmitting symbol by symbol in the local exchange phase, the source sends $s_{0}$ and $s_{1}$ simultaneously, thanks to the linear combination $s_{\text {pre }}=F_{21} s_{0}+F_{22} s_{1}$, to the cooperative node. Then the cooperative node receives

$$
y_{c_{p r e}}=h \sqrt{2 E_{L} G_{S C}} s_{p r e}+n_{c},
$$

The remaining steps are similar to the local orthogonal transmission. The destination then receives

$$
\mathbf{y}=\mathbf{G H}\left(\begin{array}{c}
\sqrt{2 E_{D}}\left(F_{11} s_{0}+F_{12} s_{1}\right) \\
g_{2} y_{c_{p r e}}
\end{array}\right)+\mathbf{G n},
$$

where $g_{2}=\frac{h^{*}}{|h|} \sqrt{\frac{2 E_{D} G_{C D}}{2 E_{L} G_{S C}|h|^{2}+N_{n_{c}}}}$.

2) Channel customizing: In this scheme, we take the channel $h$ into account by a customized channel via an equivalent transformation of the received constellation. Like in the factor multiplying scheme, two configurations will be studied regarding the local data exchange. The received signals during the precoding transmission for the two configurations are as follows. 
- Configuration 1: The equation (9) is rewritten as

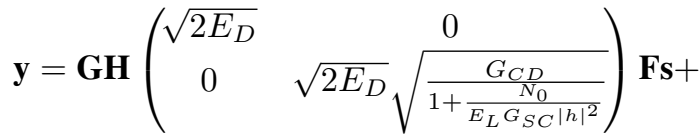

$$
\begin{aligned}
& +\left(\begin{array}{l}
H_{12}\left(g_{1} F_{21} n_{c 0}+g_{1} F_{22} n_{c 1}\right) \\
H_{22}\left(g_{1} F_{21} n_{c 0}+g_{1} F_{22} n_{c 1}\right)
\end{array}\right)+\mathbf{n}_{v} \\
& =\sqrt{2 E_{D}} \mathbf{G H}\left(\begin{array}{cc}
1 & 0 \\
0 & w_{1}
\end{array}\right) \mathbf{F s}+\mathbf{n}_{c}^{\prime}+\mathbf{n}_{v},
\end{aligned}
$$

where $w_{1}=\sqrt{\frac{G_{C D}}{1+\frac{N_{0}}{E_{L} G_{S C}|h|^{2}}}}$,

$$
\mathbf{n}_{c}^{\prime}=\left(\begin{array}{l}
H_{12}\left(g_{1} F_{21} n_{c 0}+g_{1} F_{22} n_{c 1}\right) \\
H_{22}\left(g_{1} F_{21} n_{c 0}+g_{1} F_{22} n_{c 1}\right)
\end{array}\right) .
$$

- Configuration 2: Similarly, equation (12) is rewritten as

$$
\mathbf{y}=\sqrt{2 E_{D}} \mathbf{G H}\left(\begin{array}{cc}
1 & 0 \\
0 & w_{2}
\end{array}\right) \mathbf{F s}+\left(\begin{array}{l}
H_{12} g_{2} n_{c} \\
H_{22} g_{2} n_{c}
\end{array}\right)+\mathbf{n}_{v}
$$

where $w_{2}=\sqrt{\frac{G_{C D}}{1+\frac{N_{0}}{2 E_{L} G_{S C}|h|^{2}}}}$.

Therefore, the precoding matrix is designed based on a customized channel matrix involving the contribution of both $\mathbf{H}$ and $h$ such as

$$
\mathbf{F}=\max -d_{\min }\left(\mathbf{H}\left(\begin{array}{cc}
1 & 0 \\
0 & w_{i}
\end{array}\right)\right), \quad i=1,2 .
$$

Regarding the complexity of the signal processing at the sensor nodes, thank to a limited feedback the sensor nodes are absolved from the computation. Therefore, there aren't any complexity difference between our distributed precoding and the cooperative STBC scheme. Like in other cooperative system, the main problem in our system is the desynchronization that may seriously affect the system performance [16].

\section{ENERGY MODEL}

The total energy on a wireless system consists of two elements: transmission energy and circuit consumption energy. For a same BER target, a cooperative scheme requires less transmission energy; reversely it demands more power for cooperative data exchange and circuit consumption on cooperative nodes. Certainly, for short distances, the cooperative scheme will not offer a better energy efficiency than non cooperative transmission. For longer distances, where the transmission consumption dominates the total energy, we expect that cooperative systems provide high energy efficiency. In this section we describe the energy model used to estimate the energy consumption of both cooperative and non cooperative systems.

\section{A. Circuit energy consumption model}

In a wireless system, both the transmitter and receiver possess radio frequency blocks such as filter, power amplifier, digital-to-analog converter, mixer... [2]. The circuit power consumption for each transmitter and receiver then is given by

$$
\begin{gathered}
P_{T x}=P_{D A C}+P_{m i x}+P_{f i l t}+P_{s y n} \\
P_{R x}=P_{L N A}+P_{m i x}+P_{I F A}+P_{f i l r}+P_{A D C}+P_{s y n}
\end{gathered}
$$

where $\quad P_{D A C}, P_{m i x}, P_{\text {filt }}, P_{\text {syn }}, P_{L N A}, P_{I F A}, P_{f i l r}, P_{A D C}$ denote respectively the power of digital-analog converter, mixer, filter at transmitter, synthesizer, low noise amplifier, intermediate frequency amplifier, filter at receiver and analog-digital converter. The amplifier power $P_{p a}$ for the emission relates directly to the transmission power $P_{\text {trans }}$ : $P_{p a}=(1+\alpha) P_{\text {trans }}$, where $\alpha=\frac{\xi}{\eta}-1$ with $\eta$ is the drain efficiency of the RF power amplifier and $\xi$ is the Peakto-Average Ratio (PAR) which depends on the modulation scheme and the associated constellation size [2].

\section{B. Transmission power consumption}

We suppose the radio signal is impaired by a path loss following a K-law. The required transmission power therefore is given by

$$
P_{\text {trans }}(d)=\hat{E}_{b} R_{b} \times \frac{(4 \pi d)^{K}}{G_{t} G_{r} \lambda^{2}} M_{l} N_{f},
$$

where $d$ denotes the transmission distance, $\hat{E}_{b}$ denotes the needed energy per bit for a BER target, $R_{b}$ is the bit rate, $G_{t}$ and $G_{r}$ are the antenna gains of transmission and reception side, respectively. $\lambda$ is the carrier wave length, $M_{l}$ is the link margin, $N_{f}$ is the receiver noise figure defined as $N_{f}=M_{n} / N_{0}$ with $N_{0}=-174 \mathrm{dBm} / \mathrm{Hz}$ single side thermal noise Power Spectral Density (PSD) and $M_{n}$ denotes the PSD of the total effective noise at receiver input [2].

\section{Spectral efficiency and bit rate}

The total spectral efficiency with QPSK modulation is shown in the Table I for the different schemes, cooperative and non cooperative.

TABLE I

SPECTRAL EFFICIENCY

\begin{tabular}{|c|c|c|c|c|c|}
\hline & SISO & MRC & $\begin{array}{c}\text { Distributed } \\
\text { Alamouti }\end{array}$ & $\begin{array}{c}\text { DMP } \\
\text { DF }\end{array}$ & $\begin{array}{c}\text { DMP-AF } \\
\text { config. } 2\end{array}$ \\
\hline$\eta_{S}(\mathrm{~b} / \mathrm{s} / \mathrm{Hz})$ & 2 & 2 & 1 & 1.5 & 2 \\
\hline
\end{tabular}

The bit rate in the wireless system relates directly to the spectral efficiency and the bandwidth. In addition, the bit rate $R_{b}$ of each transmission phase affects the total energy consumption as follows

$$
E_{\text {total }}=\frac{P_{c_{L}}+P_{p a_{L}}}{R_{b_{L}}}+\frac{P_{c_{D}}+P_{p a_{D}}}{R_{b_{D}}},
$$

where $P_{c_{L}}, P_{p a_{L}}$ and $P_{c_{D}}, P_{p a_{D}}$ denote the total circuit power consumption and amplifier power for local data exchange phase and precoding transmission phase, respectively. $R_{b_{L}}$ and $R_{b_{D}}$ are the bit rates of the local data exchange and the precoding transmission respectively. Normally, the bit rate in a precoding system will be twice the one of a SISO system because it transmits 2 symbols in 1 time-slot instead of only 1 symbol. However, the DMP involves two phases with different bit rates according to the considered scheme. In the local exchange phase, the Distributed Alamouti, $D M P$ $D F$, and DMP-AF-LST schemes achieve the same spectral efficiency as the SISO transmission. Whereas, with 2 modulated symbols transmitted in a precoded symbol, the DMP-AF-LPT 
represents twice the bit rate of the others. In the second phase, corresponding to the precoding transmission, all DMP schemes transmit the signal twice as fast as the non cooperative schemes (SISO and SIMO), while the Distributed Alamouti scheme achieves the same bit rate as the SISO scheme.

\section{Simulation Results}

We evaluate the schemes proposed in Section III by Monte Carlo simulations. In our system, we use the QPSK modulation, the channels $h$ and $\mathbf{H}$ are Rayleigh fading channels. We transmit 150000 frames, each frame contains 100 bits. The channel is assumed to be stable for the transmission time of one frame. The distance $d_{S C}$ is supposed to be $5 \%$ of $d_{S D}$.

In the power allocation problem, we need to minimize the BER with respect to power allocation parameter $\beta$ :

$$
\begin{gathered}
\min _{\beta} \operatorname{BER}(\beta) \\
\text { subject to } E_{L}+E_{D}=\text { const. }
\end{gathered}
$$

The BER approximation is not a simple issue in the max- $d_{\min }$ precoding system and especially complicated in the distributed $\max -d_{\min }$ precoding system. In order to allocate the power, we are currently deriving theoretically a close-form of BER in our distributed scheme. Unfortunately, this work is really a difficult problem. Since the BER approximation form is not available, the power allocation parameter $\beta$ will be found by a numerical search for the optimal power allocation (OPA). For the equal power allocation (EPA), $\beta=0.5$.

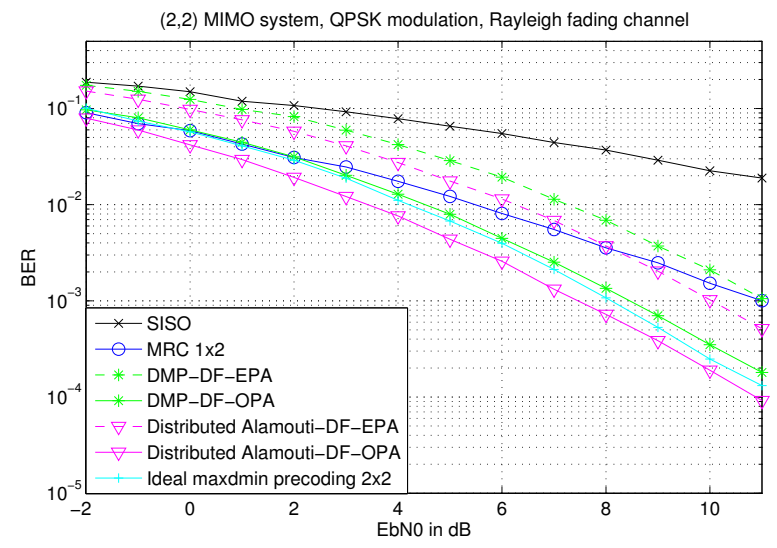

Fig. 2. BER performance comparison: DMP vs. traditional schemes

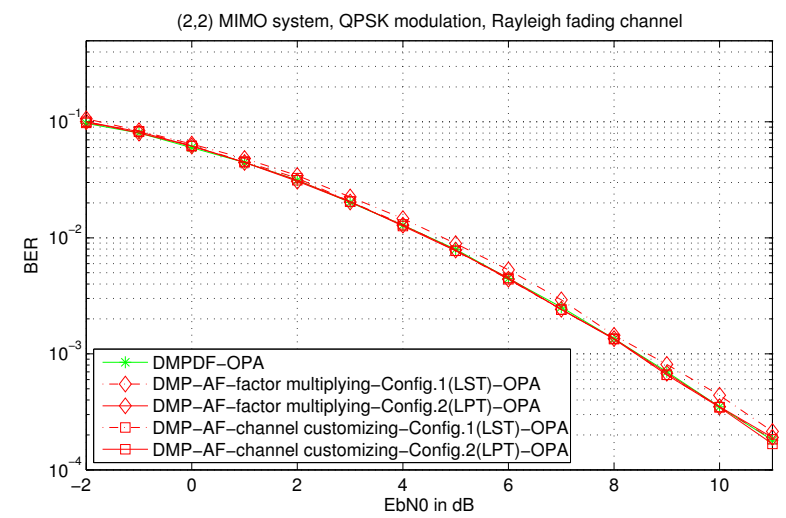

Fig. 3. BER performance comparison of DMP schemes
For QPSK modulation, $E_{b} / N_{0}=(E / 2) / N_{0}$. The BER performance comparison between various schemes is given in Figure 2. The Distributed Alamouti scheme is carried out by the Alamouti code in the virtual $2 \times 2$ MIMO system [3]. This scheme consumes 4 time-slots for transmitting 2 symbols $s_{0}, s_{1}$. The $D M P-D F$ proposes a significant transmission power reduction compared to the SISO transmission and the MRC $1 \times 2$, for the same BER target. At the BER $10^{-3}$, there is a loss of $1 \mathrm{~dB}$ in the $D M P-D F$ compared to the distributed Alamouti. We recall that they operate at different spectral efficiencies (see Table I). Figure 2 also shows the advantage of the optimal power allocation on the performance of $D M P$, the $O P A$ always provides a $3 \mathrm{~dB}$ gain compared to the EPA scheme. In Figure 3, the DMP-DF and DMP-AF schemes propose an equivalent performance. In terms of BER, even though saving 1 time slot, the DMP-AF-LPT schemes still has same performance as DMP-AF-LST. Besides, there is no difference between the $D M P-A F$ with amplifying factor and the DMP-AF with customizing channel in considering the BER.

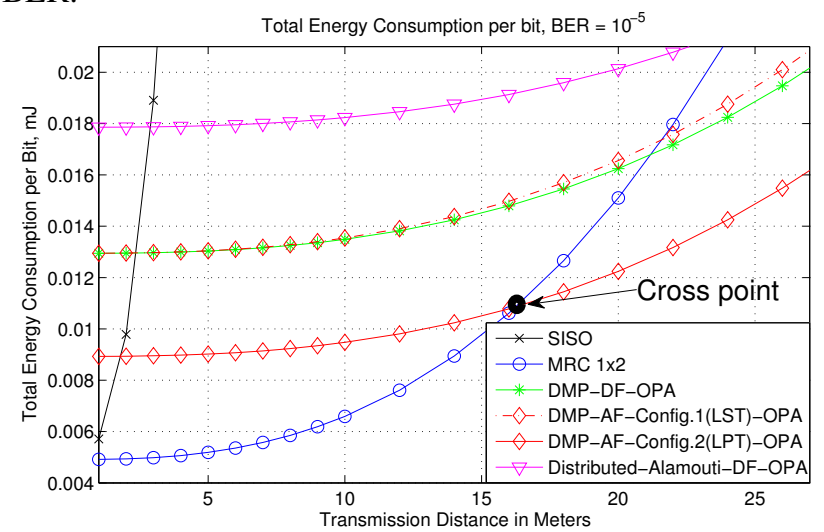

Fig. 4. Total energy consumption vs. distance $\left(10^{-5}\right.$ BER $)$

Anyhow, our final objective is the energy optimization. Thus a complete investigation of all power aspects of a wireless system must be studied to have a comprehensive view on the energy performance. We consider the energy model with the parameters given in Table II. Particularly in our system, the access point is a powerful computer plugged directly to the power source, therefore its power constraint is not taken into account. The required BER at the destination is supposed to be $10^{-5}$ and the path loss exponent is assumed to be 2.6 , for all schemes. Figure 4 shows the total energy consumption per bit for different schemes versus distances from 1 to 27 meters. There is no difference between the DMP-AF-factor multiplying and DMP-AF-channel customizing in term of BER performance and spectral efficiency, consequently we homogenize them in the energy efficiency investigation in Figure 4. By reducing 1 time slot in the local exchange phase, the DMP-AF-LPT (Config.2) always provides an energy-efficient improvement compared to the $D M P-D F$ and DMP-AF-LST (Config. 1). Moreover, regarding the distributed Alamouti scheme, even though it performs an impressive BER performance, its low spectral efficiency make it consumes more energy. On the other side, thanks to 
a good BER performance and higher spectral efficiency, the $D M P-A F-L P T$ always has a best energy efficiency compared to other $D M P$ s. For a small distance, we need only a low transmission power to obtain the targeted performance, thus the circuit consumes most of the energy. Therefore the more simple scheme will outperform the more complicated ones. The MRC $1 \times 2$ transmission takes the advantage in terms of energy efficiency in this case. In contrast, when the distance increases, the transmission energy dominates the total energy consumption. Hence, optimizing transmission energy is more valuable. If the distance is longer than 16 meters, the $D M P$ $A F-L P T$ shows the best energy efficiency.

TABLE II

PARAMETER VALUES [2]

$$
\begin{array}{c|c|c|}
f_{c}=2,5 \mathrm{GHz} & P_{\text {mix }}=30.3 \mathrm{~mW} & \eta=0.35 \\
G_{t} G_{r}=5 \mathrm{dBi} & P_{\text {filt }}=P_{\text {filr }}=2.5 \mathrm{~mW} & \\
B=10 \mathrm{kHz} & P_{D A C}=15.4 \mathrm{~mW} & P_{s y n}=50 \mathrm{~mW} \\
N_{f}=10 \mathrm{~dB} & T_{s}=\frac{1}{B} & M_{l}=40 \mathrm{~dB} \\
P_{A D C}=6.7 \mathrm{~mW} & \frac{N_{0}}{2}=-174 \mathrm{dBm} / \mathrm{Hz} & P_{L N A}=20 \mathrm{~mW}
\end{array}
$$

The path loss exponent $K=2.6$ is just an assumption. The environment can be more intricate: indoor, outdoor, crowded, or anechoic environment. Obviously, in each path loss environment, our distributed scheme responds differently. For an overall view, in Figure 5 we investigate the cross point for different path loss exponents. The Cross point (Figure 4) represents the distance at which the DMP-AF-LPT outperforms the others. We observe that when the path loss goes heavier, the $D M P$ takes the advantage for shorter distances.

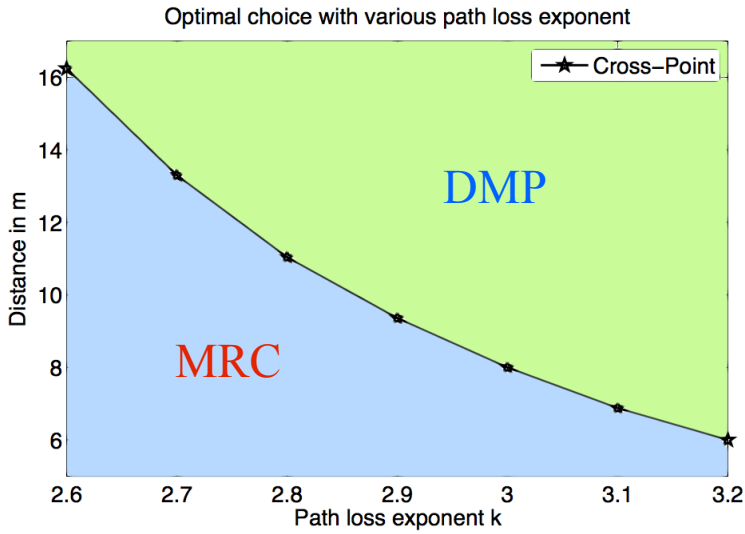

Fig. 5. Best choice point for different path loss exponents

\section{CONCLUSION}

Aiming to a high energy efficiency, we have proposed and investigated the distributed precoding schemes based on max $-d_{\text {min }}$ criterion. Two relaying types - Decode and Forward, Amplify and Forward - with different data exchanges (local successive transmission, local precoded transmission) have been defined and studied. Based on an overall energy model, we have derived their energy efficiency in WSN context. With respect to other cooperative techniques, the $D M P$ proposes a high spectral efficiency, typically doubled compared to the Distributed Alamouti one. Whatever the distance, the DMP always achieves a lower energy consumption than the Distributed Alamouti scheme. For a BER equal to $10^{-5}$ and a path loss exponent equal to 2.6, the DMP-AF-LPT achieves a lower energy consumption than the MRC $1 \times 2$ scheme for a distance between the source to access point greater than $16 \mathrm{~m}$. Moreover, when the path loss exponent increases, transmission energy becomes preponderant with respect to circuit energy and the $D M P$ takes the advantage for smaller distances, typically several meters.

\section{ACKNOWLEDGMENT}

This work has received a French state support granted to the CominLabs excellence laboratory and managed by the National Research Agency in the "Investing for the Future" program under reference $\mathrm{Nb}$. ANR-10-LABX-07-01.

\section{REFERENCES}

[1] J. N. Laneman and G. W. Wornell, "Energy-efficient antenna sharing and relaying for wireless networks," in IEEE Wireless Communications and Networking Confernce (WCNC), vol. 1, pp. 7-12, 2000.

[2] S. Cui, A. J. Goldsmith, and A. Bahai, "Energy-efficiency of MIMO and cooperative MIMO techniques in sensor networks," IEEE Journal on Selected Areas in Communications, vol. 22, no. 6, pp. 1089-1098, 2004.

[3] T.-D. Nguyen, O. Berder, and O. Sentieys, "Cooperative MIMO schemes optimal selection for wireless sensor networks," in IEEE Vehicular Technology Conference Spring (VTC-Spring), pp. 85-89, 2007.

[4] P. Stoica and G. Ganesan, "Maximum-SNR spatial-temporal formatting designs for MIMO channels," IEEE Transactions on Signal Processing, vol. 50, no. 12, pp. 3036-3042, 2002.

[5] H. Sampath, P. Stoica, and A. Paulraj, "Generalized linear precoder and decoder design for MIMO channels using the weighted MMSE criterion," IEEE Transactions on Communications, vol. 49, no. 12, pp. 2198-2206, 2001.

[6] L. Collin, O. Berder, P. Rostaing, and G. Burel, "Optimal minimum distance-based precoder for MIMO spatial multiplexing systems," IEEE Transactions on Signal Processing, vol. 52, no. 3, pp. 617-627, 2004.

[7] Q.-T. Ngo, O. Berder, B. Vrigneau, and O. Sentieys, "Minimum distance based precoder for MIMO-OFDM systems using a 16-qam modulation," in IEEE International Conference on Communications (ICC), pp. 1-5, 2009.

[8] W. W. Ho, T. Q. Quek, and S. Sun, "Distributed precoding for network MIMO," in IEEE International Conference on Communications (ICC), pp. 1-5, 2010.

[9] H.-J. Choi, S.-H. Park, S.-R. Lee, and I. Lee, "Distributed precoding techniques for weighted sum rate maximization in MIMO interfering broadcast channels," in IEEE Vehicular Technology Conference (VTC Fall), pp. 1-5, 2012.

[10] R. Zakhour and D. Gesbert, "Distributed multicell-MISO precoding using the layered virtual SINR framework," IEEE Transactions on Wireless Communications, vol. 9, no. 8, pp. 2444-2448, 2010.

[11] G. Barriac, R. Mudumbai, and U. Madhow, "Distributed beamforming for information transfer in sensor networks," in Third International Symposium on Information Processing in Sensor Networks (IPSN), pp. 81-88, 2004.

[12] V. Havary-Nassab, S. Shahbazpanahi, A. Grami, and Z.-Q. Luo, "Distributed beamforming for relay networks based on second-order statistics of the channel state information,' IEEE Transactions on Signal Processing, vol. 56, no. 9, pp. 4306-4316, 2008.

[13] G. Madi, B. Vrigneau, A.-M. Poussard, R. Vauzelle et al., "Cooperative MIMO precoders for energy-efficient transmission in wireless sensor network," in Proceedings of EusipCo, 2011.

[14] D. J. Love, R. W. Heath, V. K. Lau, D. Gesbert, B. D. Rao, and M. Andrews, "An overview of limited feedback in wireless communication systems," IEEE Journal on Selected Areas in Communications, vol. 26, no. 8, pp. 1341-1365, 2008.

[15] M. Safari and M. Uysal, "Cooperative diversity over log-normal fading channels: performance analysis and optimization," IEEE Transactions on Wireless Communications, vol. 7, no. 5, pp. 1963-1972, 2008.

[16] T.-D. Nguyen, O. Berder, and O. Sentieys, "Impact of transmission synchronization error and cooperative reception techniques on the performance of cooperative mimo systems," in IEEE International Conference on Communications (ICC), pp. 4601-4605, 2008. 